\title{
Electrical tree growth and partial discharges analyzed by fractal and correlation dimensions
}

DOI:

10.1109/CEIDP.2017.8257601

\section{Document Version}

Accepted author manuscript

Link to publication record in Manchester Research Explorer

\section{Citation for published version (APA):}

Schurch, R., Donoso, P., Aguirre, P., Cardenas, OB., Zuniga, M., \& Rowland, S. (2018). Electrical tree growth and partial discharges analyzed by fractal and correlation dimensions. In IEEE Conference on Electrical Insulation and Dielectric Phenomena (CEIDP) https://doi.org/10.1109/CEIDP.2017.8257601

\section{Published in:}

IEEE Conference on Electrical Insulation and Dielectric Phenomena (CEIDP)

\section{Citing this paper}

Please note that where the full-text provided on Manchester Research Explorer is the Author Accepted Manuscript or Proof version this may differ from the final Published version. If citing, it is advised that you check and use the publisher's definitive version.

\section{General rights}

Copyright and moral rights for the publications made accessible in the Research Explorer are retained by the authors and/or other copyright owners and it is a condition of accessing publications that users recognise and abide by the legal requirements associated with these rights.

\section{Takedown policy}

If you believe that this document breaches copyright please refer to the University of Manchester's Takedown Procedures [http://man.ac.uk/04Y6Bo] or contact uml.scholarlycommunications@manchester.ac.uk providing relevant details, so we can investigate your claim.

\section{OPEN ACCESS}




\title{
Electrical tree growth and partial discharges analyzed by fractal and correlation dimensions
}

\author{
Roger Schurch ${ }^{1}$, Pablo Donoso ${ }^{1}$, Pablo Aguirre ${ }^{2}$, O’Bryan Cardenas 2 , Marcos Zuniga ${ }^{3}$ and Simon M. Rowland ${ }^{4}$ \\ ${ }^{1}$ Department of Electrical Engineering, ${ }^{2}$ Department of Mathematics, ${ }^{3}$ Department of Electronics \\ Universidad Tecnica Federico Santa Maria, Valparaiso, Chile. \\ ${ }^{4}$ School of Electrical and Electronic Engineering, The University of Manchester, UK \\ roger.schurch@usm.cl
}

\begin{abstract}
Electrical treeing degradation is associated with partial discharge activity. Here we relate the growth of electrical trees with the correlation dimension of the reconstructed dynamic object obtained from the nonlinear time series of the partial discharges from the tree propagation, and with the box-counting fractal dimension of the resulting 3D structure of the trees. The growth of trees at 8,10 and $12 \mathrm{kV}$ has been analyzed; different PD dynamics were found, depending on both the voltage and the stage of growth. A lower fractal dimension is shown to be related to a lower correlation dimension. The results show that nonlinear time series analysis can be an alternative method of analyzing tree growth and associated partial discharges.
\end{abstract}

\section{INTRODUCTION}

Electrical trees are hollow channels of degradation in high voltage solid polymeric insulation which, over long periods of time, lead to destructive failure of the insulation [1]. They grow under partial discharge (PD) activity, and as they grow, the PD pattern evolves having a dynamic and complex behavior. Two common ways of studying treeing phenomena are analyzing the geometrical structure of trees and the PD activity during growth [2]-[7]. Commonly, 'phase-resolved PD' (PRPD) analysis, and to a lesser extent 'pulse sequence analysis' (PSA) have been used to characterize tree growth and these have been related to stages of propagation. More recently, the tool of nonlinear time series analysis and chaos theory have been explored to characterize tree growth [8], [9].

The structure of trees has been widely characterized by the tree length and fractal dimension. Fractal dimension has typically been calculated using two-dimensional (2D) images; however, the fractal dimension of 2D projected patterns differ from those of the complete three-dimensional (3D) pattern. It has recently been shown that electrical trees can be 3D imaged and the structure extensively characterized [1], [10], [11].

In this paper we correlate the stage of growth of electrical trees with the correlation dimension of the reconstructed dynamical object obtained from the nonlinear time series of the partial discharges (PD) associated with the treeing propagation, and to the box-counting fractal dimension of the resulting $3 \mathrm{D}$ structure of the mature trees. The aim is to provide an alternative method for analyzing PD and electrical trees.

\section{FRACTAL AND CORRELATION DIMENSIONS}

One of the most common ways in which fractal objects may appear is through purely geometric constructions; the Koch curve, the Sierpinski triangle or the Cantor set are classic examples [12]. However, fractal objects may also appear naturally as invariant (limit) sets of certain dynamic systems in many applications. In either case, the most common way to measure the "fractality" of these sets is to quantify how such an object "fills" the space. In this way, a fractal object will usually display a non-integer dimension typical of their fragmented, self-similar geometry. This dimension is obtained by extending the concept of dimension associated with classical Euclidean sets (lines, surfaces, volumes, etc.) to one that suits the nature of a given fractal set.

\section{A. Fractal dimension}

Several different definitions of fractal dimension and a number of methods for computing them have been devised [13]. In this paper, the term fractal dimension will refer to the "boxcounting' fractal dimension, which sometimes is also called the 'capacity dimension'. The 'box-counting' method [14] is a covering method, in which the space is divided into cubes (or squares in the 2D case) with edge length $r$. Then one counts the number of cubes (squares) $N_{(r)}$ required to cover the whole shape. If the relation

$$
N_{(r)} \propto r^{-D_{f}}
$$

is satisfied, then $D_{f}$ is the fractal dimension. The quantity $D_{f}$ is estimated from the slope of the logarithmic plot of the number of boxes $\left(N_{(r)}\right)$ against the size of the box $(r)$ that was utilised to fill the feature. Formally, the value of $\mathrm{D}_{\mathrm{f}}$ is obtained as the limit when the size of the box tends to zero [12]:

$$
D_{f}=\lim _{r \rightarrow 0} \frac{\log N_{(r)}}{-\log r}
$$

\section{B. Correlation dimension}

The concept of correlation dimension comes from the theory of dynamical systems and requires a brief introduction. Let us think of a quantity that evolves in time governed by a set of ordinary differential equations or iterations of a map, etc. We can suppose that we let the system evolve from an initial condition until we observe that in the long term the trajectory seems to "settle down" to a bounded region of phase space. This asymptotic limit set of the trajectory is called an attractor and will be denoted as $A$. In some cases, this attractor can be a very complex object with fractal properties. One refers to such an object as a chaotic or strange attractor.

However, in many experimental settings one does not know the explicit equations or evolution rule that governs the variation 
of our system in time, and yet we are still able to record and measure the quantities of interest. In such scenarios, we would like to say something useful about the fractal properties of the (unknown) subjacent dynamical system. This is possible thanks to the embedding or phase space reconstruction method [12]. Let us assume we make measurements for a single variable, say $\mathrm{x}$, in our system, which is observed in $\mathrm{N}$ occasions, resulting in a time series $\mathrm{X}=\left(\mathrm{x}_{1}, \mathrm{x}_{2}, \ldots, \mathrm{x}_{\mathrm{N}}\right)$. The idea of the reconstruction method is to consider a set of vectors

$$
\mathrm{Y}_{\mathrm{k}}=\left(\mathrm{x}_{\mathrm{k}}, \mathrm{x}_{\mathrm{k}+\mathrm{T}}, \mathrm{x}_{\mathrm{k}+2 \mathrm{~T}}, \ldots, \mathrm{x}_{\mathrm{k}+(\mathrm{m}-1) \mathrm{T}}\right)
$$

of length $m$ (also known as embedding dimension), constructed from the series $\mathrm{X}$, where we allow a time shift (also called delay) $\mathrm{T}>0$ between consecutive points. Provided $m$ is sufficiently large and $\mathrm{T}$ is chosen appropriately, and under certain technical conditions, the set of reconstructed m-dimensional points emerges as an approximation of the chaotic set $A$ in the subjacent dynamical system. The associated formal result is known as Takens' reconstruction theorem [12].

Assume we have generated $\mathrm{M}$ of such reconstructed vectors $\left\{Y_{i}, i=1, \ldots, M\right\}$ from the original series $X$ by the embedding method. We can calculate the correlation dimension of the theoretical chaotic set $A$ that contains these $m$-dimensional vectors $\mathrm{Y}_{\mathrm{i}}$. First, we define the correlation integral of the set $\left\{\mathrm{X}_{\mathrm{i}}\right\}$ as

$$
C(\epsilon)=\lim _{N \rightarrow \infty} \frac{1}{N^{2}}\left(\#(i, j) \mid i<j \text { such that }\left\|Y_{i}-Y_{j}\right\|<\epsilon\right)
$$

where $\left\|Y_{i}-Y_{j}\right\|$ denotes the Euclidean distance between the vectors $Y_{i}$ and $Y_{j}$ [12], [15]. The number $C(\varepsilon)$ can be interpreted as an average distribution of points of the set $\left\{\mathrm{Y}_{i}\right\}$ in small balls of radius $\varepsilon$. The correlation dimension of $\left\{\mathrm{Y}_{\mathrm{i}}\right\}$ is then defined as the following limit [12]:

$$
D_{c}=\limsup _{\epsilon \rightarrow 0} \frac{\log C(\epsilon)}{\log \epsilon}
$$

This definition implies that, for sufficiently small $\varepsilon$, we have

$$
\log C(\epsilon) \approx D_{c} \log (\epsilon)
$$

Hence, the correlation dimension $\mathrm{D}_{\mathrm{c}}$ can be estimated as the slope of $\log C(\varepsilon)$ vs $\log (\varepsilon)$ for small $\varepsilon$.

\section{METHODOLOGY}

The tree growth of three samples, aged at 8,10 and $12 \mathrm{kV}$, were analyzed by their tree length, fractal dimension of their 3D virtual replicas and correlation dimension of the nonlinear time series of the PD associated with the tree propagation.

\section{A. Experimental and $3 D$ models}

The samples were prepared using the conventional needleto-plane configuration with a gap of $\sim 2 \mathrm{~mm}$ between the needle tip and the bottom of the sample. The samples were made of epoxy resin (CY221-HY2966). Trees were grown in the samples by applying a $50 \mathrm{~Hz}$ voltage of 8,10 and $12 \mathrm{kV}$, where each sample was stressed for 115 minutes. The tree growth was monitored with an optical camera, and PD measurements over the entire propagation were recorded. PD were measured by a commercial system that records the apparent charge, the time and phase angle of each discharge, and separately stores the voltage waveform with a sample every $48 \mu \mathrm{s}$. The minimum apparent charge to be recorded was set to $2-3 \mathrm{pC}$ to remove background noise. The stressing time of 115 minutes was counted from the time when the tree initiated, i.e. when a small segment was noticeable by the optical camera and PD activity was detected.

After the trees were grown, the samples were 3D imaged using Synchrotron X-ray computed tomography (XCT) at the Paul Scherrer Institut [16]. 3D models (virtual replicas) of the electrical trees were generated from the experiments [1][10]. The resulting 3D models are shown in Fig. 1.

\section{B. Fractal dimension}

The fractal dimension of each electrical tree was calculated from the 3D virtual replicas. To count the number of cubes of given edge size required to cover an electrical tree, an open access Matlab code was used [17]. The input of the algorithm was the electrical tree, represented by a volumetric matrix of the 3D image and the output was a matrix with the pairs $(r, N)$ resulting from the counting algorithm. The $\mathrm{D}_{\mathrm{f}}$ value reported here is the slope of the linear regression considering only the three smallest cube sizes, a criterion that is close to the formal definition of equation 2 [18].

\section{Correlation dimension}

The correlation dimension associated with the partial discharges was obtained in two steps. All the algorithms described below were implemented in Python. First, we reconstructed the subjacent phase space from time series of the PD amplitude (apparent charge) using Takens' method. We used the criterion from Fraser and Swinney [19] and took the first local minimum of the mutual information to estimate an optimal delay parameter $T$. We used the nearest false neighbor strategy [20] to find the embedding dimension $m$. To ensure that almost all variability in the data is due to the deterministic nonlinear features of the system and not to random noise every time series passed a test for determinism.

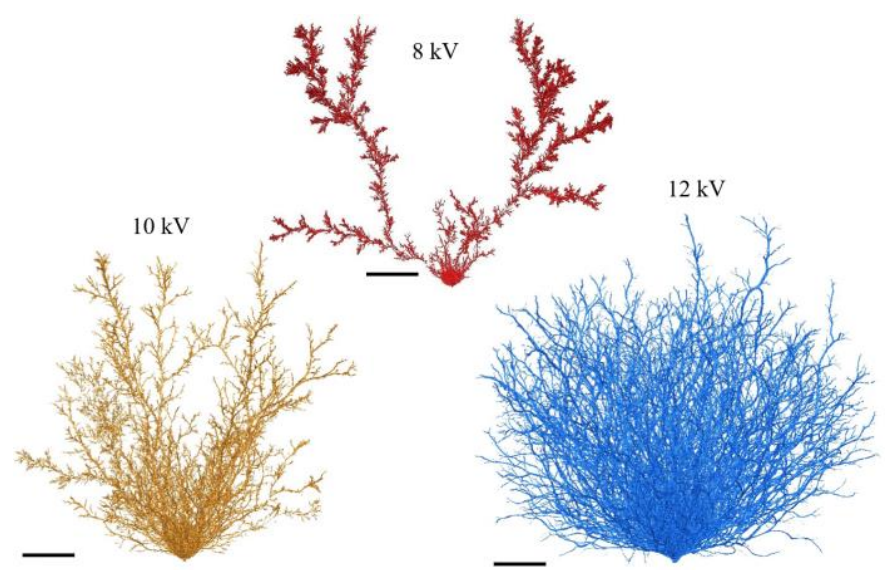

Fig. 1. 3D renderings of samples aged at $8, \overline{10}$ and $12 \mathrm{kV}$. Scale bar: $200 \mu \mathrm{m}$. 
The second step in our procedure was the computation of the correlation dimension of the $M$ reconstructed vectors with a modified Grassberger and Procaccia algorithm (GPA) [15]. The modification consists on applying the GPA to a subset of points in every reconstructed trajectory. Points in this subset are randomly chosen until the average distance of these points to the rest is less than a given threshold or representativeness index $\alpha$. The smaller the chosen $\alpha$, the more points that have to be considered to apply the GPA. This modified GPA was tested with several objects of known correlation dimension, namely, a 2D square, a 3D cube, and the famous Lorenz attractor. In all cases, the error was below $3 \%$.

\section{RESULTS AND DISCUSSION}

The continuous PD recordings of the samples are depicted in Fig. 2, where the time series of PD amplitude is shown over the 115 minutes of tree growth. This stressing time was chosen to avoid breakdown, so a mature 3D replica of tree could be obtained, as shown in Fig. 1. For the sample aged at $8 \mathrm{kV}$ no PD were detected after 71 minutes, though the tree continued growing. It is apparent that something similar was going to happen for the sample aged at $10 \mathrm{kV}$, because from 112 minutes, only low levels of PD near to the noise level were detected. It is seen that the tree growth behavior for samples aged at 8 and $10 \mathrm{kV}$ is almost the same during the first hour of propagation. It can be also observed that different PD behaviors, both in amplitude and pattern as function of time, were obtained (Fig. 2 ) when different voltages were applied and, as expected, this resulted in different tree shapes: from branch-pine type at $8 \mathrm{kV}$ to bush-type at $12 \mathrm{kV}$ (Fig. 1).

To analyze the progression of the correlation dimension with the tree growth, subseries (PD intervals) were selected; these are the darker bands shown in the PD time series of Fig. 2. Each selected time interval is chosen to be at least 10 seconds long. Moreover, the product of each interval time and the number of points it contains is at least 100,000 second-points. Using this criterion, the number of points of each selected interval (band) was between 2,000 to 28,000 points, which was sufficient to estimate the dynamic properties. The first interval was after two minutes of propagation and the subsequent intervals were every 10 minutes; however, the last interval was arbitrarily placed near to the end of the series, for a better correlation with the fractal dimension of the 3D replica. To correlate with the progression of the tree, the tree length was taken from the optical images and measured as the furthest tree extent from the needle tip.

The results of tree length and correlation dimension for the samples aged at 8,10 and $12 \mathrm{kV}$ are shown in Fig. 2. There was no clear tendency or behavior of the correlation dimension for the sample aged at $8 \mathrm{kV}$. For the sample aged at $10 \mathrm{kV}$, the correlation dimension increased when both the PD amplitude and the tree growth rate increased, which was after 62 minutes. An exception to this occurred for the last interval, when the correlation dimension sharply dropped; while the reasons for this are to be investigated, the decrease of the correlation dimension coincides with lower PD activity: 2,765 PD/s in the interval at 102 minutes against $1,003 \mathrm{PD} / \mathrm{s}$ at 111 minutes, and also a decrease in PD amplitude. The PD dynamics terminate in a different resting state, reflected by the increasingly low activity towards the end of the experiment. For the sample aged at
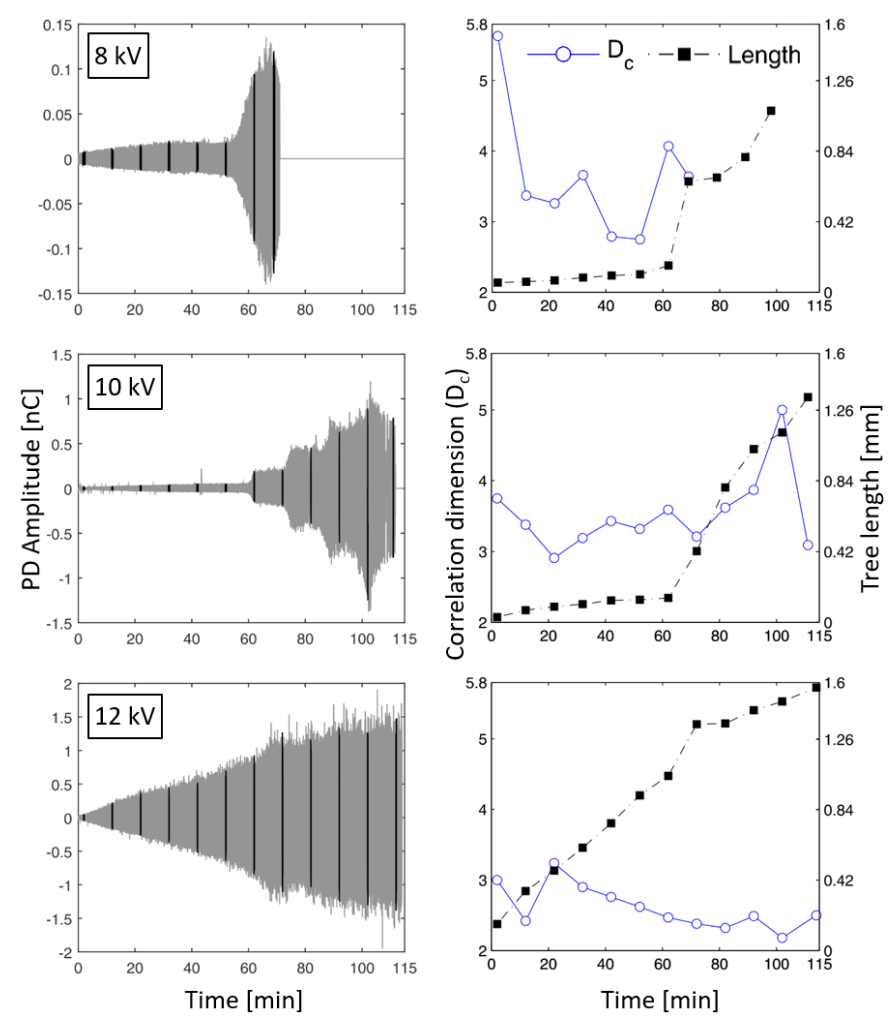

Fig. 2. Left: Time series of PD amplitude for samples aged at 8 (top), 10 (middle) and $12 \mathrm{kV}$ (bottom). Right: Correlation dimension and tree length as a function of treeing time.

$12 \mathrm{kV}$, there was a clear decreasing tendency of the correlation dimension; this behavior has been reported in [8] for treeing at 9.2 and $12.2 \mathrm{kV}$. However, it is different to the results obtained in [9] where samples aged at 11 and $15 \mathrm{kV}$ showed an initial increase of the correlation dimension and then became stable or increased gradually, respectively. Unlike the sample aged at $10 \mathrm{kV}$, the correlation dimension for the sample aged at $12 \mathrm{kV}$ decreased while the PD magnitude was continuously increasing. Moreover, the correlation dimension of the sample aged at $12 \mathrm{kV}$ was in every interval (except one) lower than the other samples.

The comparison between the correlation dimension obtained from the time series of PD amplitude and the box-counting fractal dimension of the 3D virtual replica of the resulting trees is shown in Table 1. As typically the fractal dimension is calculated from 2D optical images, the fractal dimension from 2D projected images was added to the table, for reference. The 2D images were obtained by projecting the 3D virtual replica onto the two orthogonal planes that are parallel to the tree growth direction. Then, the fractal dimension of these two images were averaged and are the values reported in Table 1 as '2D projection'. The values of correlation dimension were calculated using the complete time series, since in theory, the shape of the resulting electrical tree should be mainly related to the PD dynamics of the entire tree propagation process. This calculation was very demanding in computing resources; e.g. for the sample aged at $12 \mathrm{kV}$ more than 9 million of PD were used for the calculation. For the sample aged at $8 \mathrm{kV}$, the correlation dimension value is not presented, since no PD activity was detected after 71 minutes, as explained above. 
Table 1. Fractal dimension $\left(\mathrm{D}_{\mathrm{f}}\right)$ of the $3 \mathrm{D}$ tree and of the $2 \mathrm{D}$ projected images, and correlation dimension $\left(\mathrm{D}_{\mathrm{c}}\right)$ of the time series of PD amplitude.

\begin{tabular}{|l|c|c|c|}
\cline { 2 - 4 } \multicolumn{1}{c|}{ Sample } & $\mathbf{8 k V}$ & $\mathbf{1 0 k V}$ & $\mathbf{1 2 k V}$ \\
\hline $\mathrm{D}_{\mathrm{f}}$ (3D virtual replica) & 1,86 & 1,96 & 2,17 \\
\hline $\mathrm{D}_{\mathrm{f}}$ (2D projection) & 1,62 & 1,69 & 1,75 \\
\hline $\mathrm{D}_{\mathrm{c}}$ (serie complete) & - & 1,74 & 3,78 \\
\hline
\end{tabular}

Comparing the samples aged at 10 and $12 \mathrm{kV}$, it can be observed that the lower fractal dimension 1.96 of sample aged at $10 \mathrm{kV}$ was associated with the lower correlation dimension of 1.74 compared to 2.17 and 3.78 respectively, for the sample aged at $12 \mathrm{kV}$ if the data of the entire time series is considered.

\section{CONCLUSIONS}

This paper has presented an analysis of electrical tree propagation in epoxy resin samples aged at 8,10 and $12 \mathrm{kV}$ using nonlinear time series analysis. Tree growth was analyzed by relating tree length and the correlation dimension of the reconstructed dynamical object obtained from the PD amplitude time series measured during tree growth. It was found that at 10 $\mathrm{kV}$, when the tree growth rate increased the correlation dimension also increased. However, for the sample aged at $12 \mathrm{kV}$ the correlation dimension had a decreasing tendency during most of the growth.

The box-counting fractal dimension of the 3D virtual replicas of the trees in their last stages of growth were compared to the correlation dimension considering the entire tree growth time series. A lower fractal dimension was related to a lower correlation dimension. The PD dynamics change with different stressing voltages and with the stage of tree growth; therefore, nonlinear time series analysis can contribute to the complex analysis of tree propagation and corresponding degradation of high voltage polymeric insulation.

\section{ACKNOWLEDGMENT}

The authors thank Universidad Tecnica Federico Santa Maria for the 2016 multi-disciplinary project grant: 216.22.2. PA was partially supported by Proyecto Fondecyt Iniciación 11150306. SMR is grateful to EPSRC, UK for support through the project 'HubNet Research Leadership and Networking for Energy Networks EP/N030028/1. We also acknowledge the time granted on the TOMCAT beamline (ID 20140325) by the Paul Scherrer Institut, the Swiss Light Synchrotron.

\section{REFERENCES}

[1] R. Schurch, S. M. Rowland, R. S. Bradley, and P. J. Withers, "Imaging and analysis techniques for electrical trees using X-ray computed tomography," IEEE Trans. Dielectr. Electr. Insul., vol. 21, no. 1, pp. 53-63, 2014.

[2] J. V Champion, S. J. Dodd, and J. M. Alison, "The correlation between the partial discharge behaviour and the spatial and temporal development of electrical trees grown in an epoxy resin," J. Phys. D. Appl. Phys., vol. 29, no. 10, p. 2689, 1996.
[3] Suwarno and et al., "Partial discharges due to electrical treeing in polymers: phase-resolved and time-sequence observation and analysis," J. Phys. D. Appl. Phys., vol. 29, no. 11, p. 2922, 1996.

[4] M. D. Noskov, M. Sack, A. S. Malinovski, and A. J. Schwab, "Measurement and simulation of electrical tree growth and partial discharge activity in epoxy resin," J. Phys. D. Appl. Phys., vol. 34, no. 9, p. 1389, 2001.

[5] F. Guastavino and B. Cerutti, "Tree growth monitoring by means of digital partial discharge measurements," IEEE Trans. Dielectr. Electr. Insul., vol. 10, no. 1, pp. 65-72, 2003.

[6] R. Vogelsang, B. Fruth, T. Farr, and K. Fröhlich, "Detection of electrical tree propagation by partial discharge measurements," Eur. Trans. Electr. Power, vol. 15, pp. 271-284, 2005.

[7] S. J. Dodd, N. M. Chalashkanov, and J. C. Fothergill, "Partial discharge patterns in conducting and non-conducting electrical trees," in Solid Dielectrics (ICSD), 2010 10th IEEE International Conference on, 2010, pp. $1-4$.

[8] L. Barbieri, A. Villa, and R. Malgesini, "A step forward in the characterization of the partial discharge phenomenon and the degradation of insulating materials through nonlinear analysis of time series," IEEE Electr. Insul. Mag., vol. 28, no. 4, pp. 14-21, 2012.

[9] X. Chen, Y. Xu, and X. Cao, "Nonlinear time series analysis of partial discharges in electrical trees of XLPE cable insulation samples," IEEE Trans. Dielectr. Electr. Insul., vol. 21, no. 4, pp. 1455-1461, 2014.

[10] R. Schurch, S. M. Rowland, R. S. Bradley, and P. J. Withers, "Comparison and Combination of Imaging Techniques for Three Dimensional Analysis of Electrical Trees," IEEE Trans. Dielectr. Electr. Insul., vol. 22, pp. 709-719, 2015

[11] S. M. Rowland, R. Schurch, M. Pattouras, and Q. Li, "Application of FEA to image-based models of electrical trees with uniform conductivity," IEEE Trans. Dielectr. Electr. Insul., vol. 22, pp. 1537 1546,2015

[12] H. Broer and F. Takens, "Dynamical systems and chaos, vol. 172 of Applied Mathematical Sciences." Springer, New York, 2011.

[13] H. Takayasu, Fractals in the physical sciences. Manchester University Press, 1990.

[14] K. Kudo, "Fractal analysis of electrical trees," IEEE Trans. Dielectr. Electr. Insul., vol. 5, no. 5, pp. 713-727, 1998.

[15] P. Grassberger and I. Procaccia, "Measuring the strangeness of strange attractors," Phys. D Nonlinear Phenom., vol. 9, no. 1-2, pp. 189-208, 1983.

[16] R. Schurch, S. M. Rowland, and R. S. Bradley, "Partial discharge energy and electrical tree volume degraded in epoxy resin," in IEEE Conf. Electr. Insul. Dielectr. Phenom. (CEIDP), pp. 820-823, 2015.

[17] F. Moisy, "Computing a fractal dimension with Matlab: 1D, 2D and 3D Box-counting." [Online]. Available: http://www.mathworks.co.uk/matlabcentral/fileexchange/13063boxcount/content/boxcount/html/demo.html.

[18] R. Schurch, C. González, P. Aguirre, M. Zuniga, S. M. Rowland, and I. Iddrissu, "Calculating the fractal dimension from 3D images of electrical trees," in Int'l Symp. High Voltage Engineering (ISH), 2017.

[19] A. M. Fraser and H. L. Swinney, "Independent coordinates for strange attractors from mutual information," Phys. Rev. A, vol. 33, no. 2, p. 1134, 1986

[20] M. B. Kennel, R. Brown, and H. D. I. Abarbanel, "Determining embedding dimension for phase-space reconstruction using a geometrical construction," Phys. Rev. A, vol. 45, no. 6, p. 3403, 1992. 